\title{
Oxygen therapy for interstitial lung disease: a systematic review
}

\author{
Emily C. Bell ${ }^{1}$, Narelle S. Cox ${ }^{1,2,3}$, Nicole Goh ${ }^{3,4,5}$, Ian Glaspole ${ }^{5,6}$, \\ Glen P. Westall ${ }^{5,6}$, Alice Watson ${ }^{2}$ and Anne E. Holland ${ }^{2,3,7}$
}

Affiliations: ${ }^{1}$ Dept of Physiotherapy, Monash University, Clayton, Australia. ${ }^{2}$ Dept of Physiotherapy, La Trobe University, Melbourne, Australia. ${ }^{3}$ Institute for Breathing and Sleep, Melbourne, Australia. ${ }^{4}$ Respiratory and Sleep Medicine, Austin Health, Heidelberg, Australia. ${ }^{5}$ General Respiratory and Transplant, Alfred Health, Melbourne, Australia. ${ }^{6}$ Dept of Medicine, Monash University, Clayton, Australia. ${ }^{7}$ Dept of Physiotherapy, Alfred Health, Melbourne, Australia.

Correspondence: Anne E. Holland, La Trobe University Clinical School, Level 4, The Alfred Centre, 99 Commercial Rd, Melbourne, 3004, Australia. E-mail: a.hollanddalfred.org.au

@ERSpublications

Oxygen did not improve dyspnoea during exercise in ILD, but exercise capacity was increased http://ow.ly/wu8c307iGaC

Cite this article as: Bell EC, Cox NS, Goh N, et al. Oxygen therapy for interstitial lung disease: a systematic review. Eur Respir Rev 2017; 26: 160080 [https://doi.org/10.1183/16000617.0080-2016].

ABSTRACT This review aims to establish the impact of oxygen therapy on dyspnoea, health-related quality of life (HRQoL), exercise capacity and mortality in interstitial lung disease (ILD).

We included studies that compared oxygen therapy to no oxygen therapy in adults with ILD. No limitations were placed on study design or intervention type. Two reviewers independently evaluated studies for inclusion, assessed risk of bias and extracted data. The primary outcome was dyspnoea.

Eight studies evaluated the acute effects of oxygen $(n=1509)$. There was no effect of oxygen therapy on modified Borg dyspnoea score at end exercise (mean difference (MD) -0.06 units, 95\% CI $-0.24-0.13$; two studies, $\mathrm{n}=27$ ). However, effects on exercise outcomes consistently favoured oxygen therapy. One study showed reduction in dyspnoea at rest with oxygen in patients who were acutely unwell (MD visual analogue scale $30 \mathrm{~mm}$ versus $48 \mathrm{~mm}, \mathrm{p}<0.05 ; \mathrm{n}=10)$. Four studies of long-term oxygen therapy $(\mathrm{n}=2670)$ had high risk of bias and no inferences could be drawn.

This systematic review showed no effects of oxygen therapy on dyspnoea during exercise in ILD, although exercise capacity was increased. Future trials should evaluate whether acute improvements in exercise capacity with oxygen can be translated into improved physical activity and HRQoL.

\section{Introduction}

The interstitial lung diseases (ILDs) are characterised by scarring and fibrosis of lung tissue with varying degrees of hypoxaemia. Common symptoms include severe cough and dyspnoea. Despite recent advances in pharmacotherapy, many patients with ILD have limited treatment options [1]. Oxygen therapy is commonly prescribed for people with ILD, with the aim of reducing breathlessness and increasing physical capacity through improved gas exchange. Despite its frequent use there is a lack of evidence supporting the effectiveness of oxygen therapy in ILD. A previous systematic review included only one small study of long-term oxygen therapy (LTOT) that remains unpublished and which concluded there was no effect on survival [2]. However, there is a well-accepted relationship between use of LTOT and improved health

This article has supplementary material available from err.ersjournals.com

Received: Aug 162016 | Accepted after revision: Nov 202016

Conflict of interest: None declared.

Provenance: Submitted article, peer reviewed.

Copyright $\odot$ ERS 2017. ERR articles are open access and distributed under the terms of the Creative Commons Attribution Non-Commercial Licence 4.0. 
outcomes in people with chronic obstructive pulmonary disease (COPD) [3, 4], raising the possibility that LTOT could have similar effects in ILD. A recent review of ambulatory oxygen therapy in ILD excluded several trials that examined the effects of oxygen during exercise testing in ILD, such that no conclusions could be drawn regarding its impact on symptoms or exercise capacity [5]. The aim of this review was to establish the impact of oxygen therapy on dyspnoea, health-related quality of life (HRQoL), exercise capacity and mortality in ILD.

\section{Methods}

As few studies were expected, controlled, noncontrolled and crossover trials were included. Case studies were excluded. Exclusions were studies not written in English and mixed populations (e.g. ILD and COPD), where data from the ILD subgroup were not reported separately. Only studies published in the peer-reviewed literature were included; abstracts were not included.

Individuals aged $>18$ years with any type of ILD were included, regardless of disease severity. Diagnosis of ILD was made according to the investigators' definition.

All types of oxygen therapy were included. This included but was not limited to long-term oxygen therapy (LTOT), defined as prescription of oxygen for $\geqslant 15 \mathrm{~h}$ per day; short-term oxygen therapy, including but not limited to ambulatory oxygen therapy, short-burst oxygen therapy, nocturnal oxygen therapy and oxygen therapy during exercise or exercise training. Results for each type of oxygen therapy were analysed separately.

\section{Outcomes}

The primary outcome measure was dyspnoea, measured using any validated tool. Secondary outcomes included HRQoL measured by disease-specific or generic questionnaires; assessment of mood disturbance, such as anxiety or depression; functional or maximal exercise capacity, assessed by laboratory-based or field exercise tests; physical activity, using subjective or objective assessment tools; need for lung transplantation; and mortality.

\section{Data collection and analysis}

Databases searched were Medline, Embase and Cochrane, from inception until March 2016. Detailed search strategies are listed in the online supplementary material.

Search results were reviewed by title and abstract by two reviewers independently. Results were classified as "include", "unclear" or "exclude". All articles deemed "include" or "unclear" were retrieved in full text and assessed by the two reviewers using a standardised data extraction form. Disagreement was resolved by consensus. Reviewers independently assessed each study for risk of bias according to the Cochrane Handbook for Systematic Reviews of Interventions [6] across the domains of selection bias, performance bias, detection bias, attrition bias and reporting bias.

Where data were clinically homogenous, a pooled quantitative analysis was performed using Review Manager 5 software (http://community.cochrane.org/tools/review-production-tools/revman-5). Only randomised trials were considered for meta-analysis. A fixed or random effects model was used, depending on assessment of heterogeneity.

Continuous variables were expressed as weighted mean difference with $95 \%$ confidence interval or as a standardised mean difference for outcomes that could not be aggregated into a common unit of measure. Generic inverse variance methods were used for crossover trials, where mean difference between groups and standard error of the difference between groups were calculated [6]. Where data were available in crossover trials, the mean difference between oxygen and air and its 95\% confidence interval were calculated using methods for paired data [7]. Where data could not be pooled due to study design or clinical heterogeneity, a narrative review was performed.

\section{Results}

3285 articles were identified from the search (figure 1). After record screening, 69 articles were identified to be reviewed as full text. Seven additional articles were found by reviewing reference lists. A total of 13 reports of 12 studies [8-20] met the inclusion criteria.

\section{Trial characteristics}

There were no randomised controlled parallel group trials (RCTs) in the 12 included studies (online supplementary table S1). Short-term oxygen therapy was assessed in nine reports $[8-15,20]$, two of which appeared to be the same study [8,9], and LTOT in four studies [16-19]. Seven (86\%) out of eight studies of short-term oxygen therapy assessed its effects during an acute bout of exercise. A single study assessed the effect of supplemental oxygen on dyspnoea at rest [10]. No studies evaluated nocturnal oxygen 


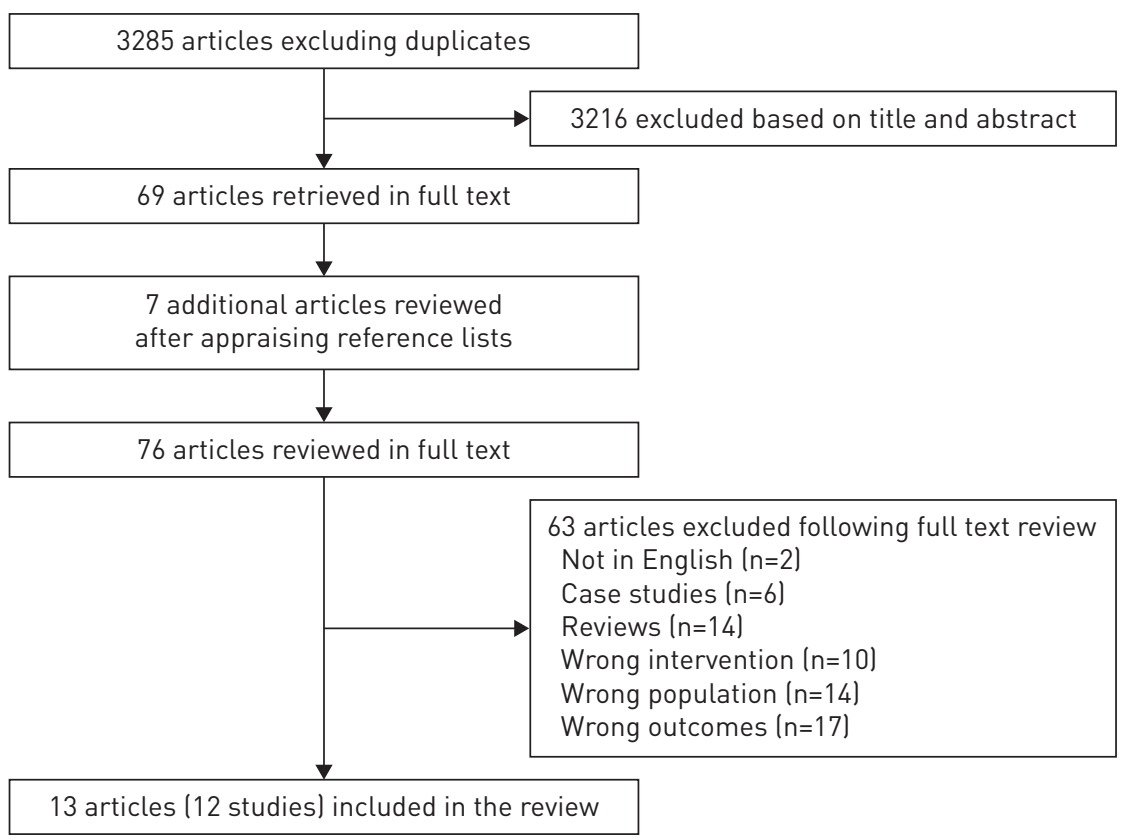

FIGURE 1 Preferred Reporting Items for Systematic Reviews and Meta-Analyses (PRISMA) diagram of study selection.

therapy, ambulatory oxygen therapy or oxygen therapy during exercise training. There were no randomised studies of LTOT. Three studies of short-term oxygen therapy were randomised crossover trials $[10,11,15]$. Six studies used retrospective data $[13,14,16-18,20]$.

\section{Participants}

4185 individuals with ILD were included. Sample sizes ranged from seven [11] to 2117 participants [17]. Mean age of participants ranged from 47 years [9] to 74 years [15]. The percentage of male participants ranged from 55\% [17] to $94 \%$ [9]. Participants generally had moderate to severe disease, with mean forced vital capacity ranging from $58 \%$ predicted [13] to $72 \%$ pred [12] and diffusing capacity of the lung for carbon monoxide ranging from $26 \%$ pred [13] to $57 \%$ pred [15].

\section{Short-term supplemental oxygen}

Studies of short-term supplemental oxygen were compared to control groups administered medical air $[8-13,15]$ or the participants' usual dosage of oxygen $[14,20]$ (range $0-6 \mathrm{~L} \cdot \mathrm{min}^{-1}$ ). Trials of short-term oxygen during acute bouts of exercise were undertaken using oxygen flow rates of $4-7 \mathrm{~L} \cdot \mathrm{min}^{-1}[12,15]$ or using oxygen prescribed at an "optimal" level (maintaining arterial oxygen saturation measured by pulse oximetry $\geqslant 90 \%$ ) [14]. Exercise test conditions utilised for the assessment of short-term oxygen were 6-min walk test $(6 \mathrm{MWT})[13-15,20]$ or laboratory based cardiopulmonary exercise testing (CPET) $[8,9,11,12]$.

\section{LTOT}

Four studies assessing LTOT in ILD included 2670 individuals [16-19]. Two uncontrolled studies reported survival rates over $\geqslant 12$ months $[16,17]$. Another retrospective review $(n=487)$ compared survival in those who were recommended oxygen therapy and those who were not, with follow-up of up to 4 years [18]. One uncontrolled study evaluated HRQoL at baseline and $\geqslant 1$ year later in 46 individuals with idiopathic pulmonary fibrosis (IPF), and reported its relationship to oxygen use [19].

\section{Risk of bias in included studies}

Only three of the included studies had random allocation, all of which randomised the treatment order of short-term oxygen therapy versus control in crossover trials $[10,11,15]$. One study had random allocation for the endurance test but not CPET [9]. Overall, studies scored poorly in performance and detection bias due to lack of blinding. Studies generally scored well in the domains of attrition and reporting. A summary of the risk of bias is presented in figure 2 and a full description is given in online supplementary table S2. 


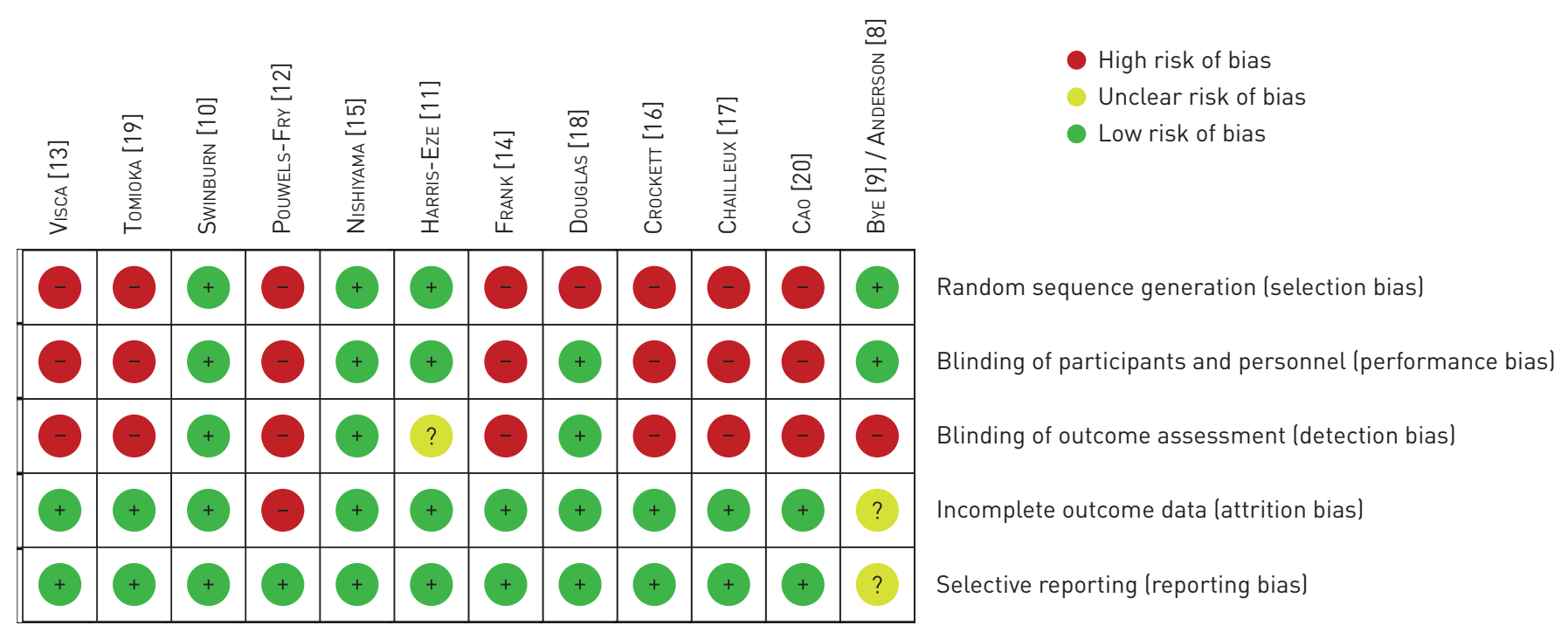

FIGURE 2 Summary of risk of bias assessment.

\section{Effects of interventions \\ Short-term supplementary oxygen \\ Primary outcome}

Six studies of short-term supplemental oxygen reported outcomes for dyspnoea, five of which were conducted during exercise and one at rest (online supplementary table S3) [10, 11, 13-15, 20]. Dyspnoea was commonly measured using the modified Borg scale $(n=5)$; however not all studies were suitable for meta-analysis due to variation in methodology, particularly lack of randomisation of treatment order. A single study assessing resting dyspnoea in hospital inpatients used a visual analogue scale (VAS) [10].

A meta-analysis was performed with two placebo-controlled studies that employed a random order of oxygen versus air during an acute bout of exercise $[11,15]$. This comparison demonstrated no effect of oxygen therapy on dyspnoea, with overall mean $(95 \% \mathrm{CI})$ difference of -0.06 units $(-0.24-0.13)$ on the modified Borg scale at the end of exercise (figure 3). Two retrospective studies without randomised ordering showed inconsistent results of oxygen on Borg dyspnoea at the end of the exercise test, with one reporting no difference [14] and the other reporting a mean reduction in Borg dyspnoea of one point $(p<0.00001)$ [13]. A retrospective review of 3126 patients with fibrotic ILD found that patients who used oxygen during a 6MWT had significantly more dyspnoea at the end of the test than those who did not use oxygen, regardless of whether or not they desaturated during the test (desaturators: oxygen versus non-oxygen users modified Borg score $4.3 \pm 2.0$ versus $3.4 \pm 1.7$ units, $\mathrm{p}<0.0001$; non-desaturators: oxygen versus non-oxygen users $3.4 \pm 1.9$ versus $2.4 \pm 1.6$ units, $\mathrm{p}<0.0001$ ) [20]. In patients with ILD who were hospitalised, breathing $28 \%$ oxygen via a face mask significantly decreased dyspnoea on a 100-mm VAS (mean $30.2 \mathrm{~mm}$ versus $48.1 \mathrm{~mm}, \mathrm{p}<0.05$ ) [10].

\section{Secondary outcomes}

The effect of supplemental oxygen on peak work capacity during CPET was investigated in three crossover trials (four reports) $[8,9,11,12]$. In the only randomised trial, supplemental oxygen significantly improved peak work capacity by a mean (95\% CI) 17 (10.60-23.40) W compared to medical air (online supplementary table S4) [11]. This is consistent with the results of the non-randomised studies [8, 9, 12], although only one [12] reached statistical significance.

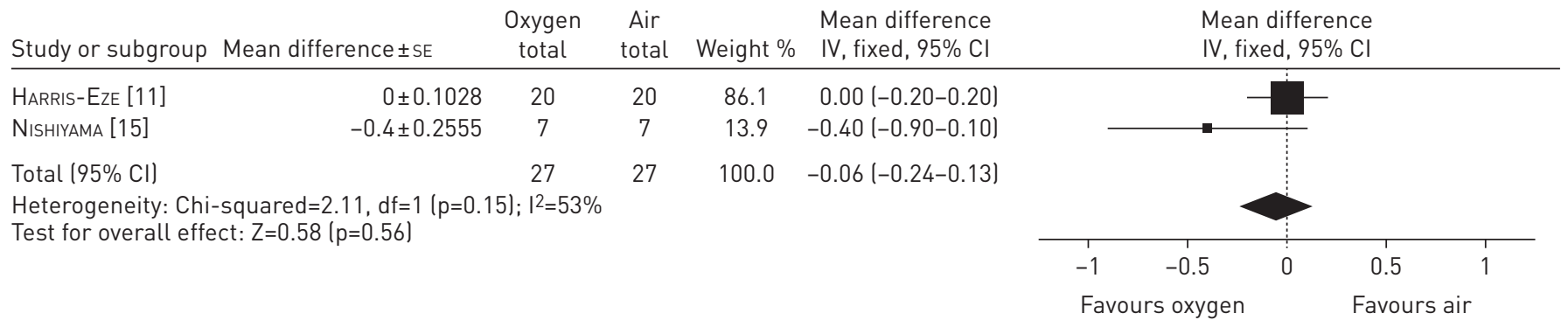

FIGURE 3 Effect of oxygen compared to air on modified Borg scale in randomised crossover trials. 
Two studies reported measurement of peak oxygen uptake $\left(\mathrm{VO}_{2}\right.$ peak) (online supplementary table S5) $[9,11]$. One trial with randomised order reported significantly greater $\mathrm{VO}_{2}$ peak when breathing oxygen compared to medical air $\left(1.58 \mathrm{~L} \cdot \mathrm{min}^{-1}\right.$ versus $\left.1.32 \mathrm{~L} \cdot \mathrm{min}^{-1}, \mathrm{p}<0.05\right)$ [11]. The remaining trial [9] did not report the data for $\mathrm{VO}_{2}$ peak while breathing oxygen.

One study (two reports) investigated the effect of short-term supplemental oxygen on cycle endurance time $[8,9]$, assessed at $80 \%$ of maximal work-rate using $60 \%$ oxygen versus air. Mean (95\% CI) improvement in endurance time when breathing $60 \%$ oxygen was $6(3.64-8.26)$ min (online supplementary table S6).

The effect of supplemental oxygen on 6-min walking distance (6MWD) was investigated in four studies (online supplementary table S7) [13-15, 20]. One placebo-controlled crossover trial with randomised order of test conditions reported no significant improvement in 6MWD using oxygen compared to air (mean (95\% CI) improvement $13(-24-50) \mathrm{m})$ [15]. In contrast, the two retrospective studies with nonrandomised order of test conditions and no participant blinding reported marked improvements in 6MWD in favour of oxygen of $81.2 \mathrm{~m}(\mathrm{p}<0.01)$ [14] and $30.9 \mathrm{~m}(\mathrm{p}<0.00001)$ [13]. A retrospective study found that those patients who used oxygen during a 6MWT had a lower average 6MWD than those who did not, regardless of whether they desaturated during the test (desaturators: oxygen versus non-oxygen users 6MWD $320 \pm 117 \mathrm{~m}$ versus $431 \pm 113 \mathrm{~m}, \mathrm{p}<0.0001$; non-desaturators: oxygen versus non-oxygen users $332 \pm 102 \mathrm{~m}$ versus $435 \pm 96 \mathrm{~m}, \mathrm{p}<0.0001$ ) [20].

The outcomes of HRQoL, mood disturbance, need for lung transplantation or survival were not reported in any of the studies of short-term supplemental oxygen.

A summary of the effect of short-term oxygen on outcomes in randomised trials is presented in table 1.

LTOT

Primary outcome

Dyspnoea was not reported in any of the studies of LTOT.

\section{Secondary outcomes}

Survival was reported in three studies of LTOT (online supplementary table S8) [16-18]. All of these studies demonstrated poor survival rates for those with ILD. One study reported that $50 \%$ of patients with fibrosis or pneumoconiosis died within 1 year of commencing home oxygen or ventilation [17]. As these trials were not randomised and two did not include comparison groups [16, 17], it was not possible to directly evaluate the impact of LTOT on survival. However, an indirect comparison was made in one study of 487 individuals with IPF, demonstrating an increased risk of death in those who had been recommended oxygen therapy compared to those who had not (relative risk 2.0, 95\% CI 1.5-2.6) [18]. This association was no longer present in a multivariate analysis controlling for age, sex, baseline respiratory function and disease progression on respiratory function tests [18].

HRQoL was reported in a single study of LTOT [19]. Participants with ILD using LTOT demonstrated a substantial decline in five out of eight categories on the 36-item short-form survey (SF-36). Participants who did not require LTOT had a better HRQoL over the duration of the study. This study did not control for the effects of disease severity on HRQoL.

TABLE 1 Summary of the effects of short-term oxygen compared to air in randomised trials

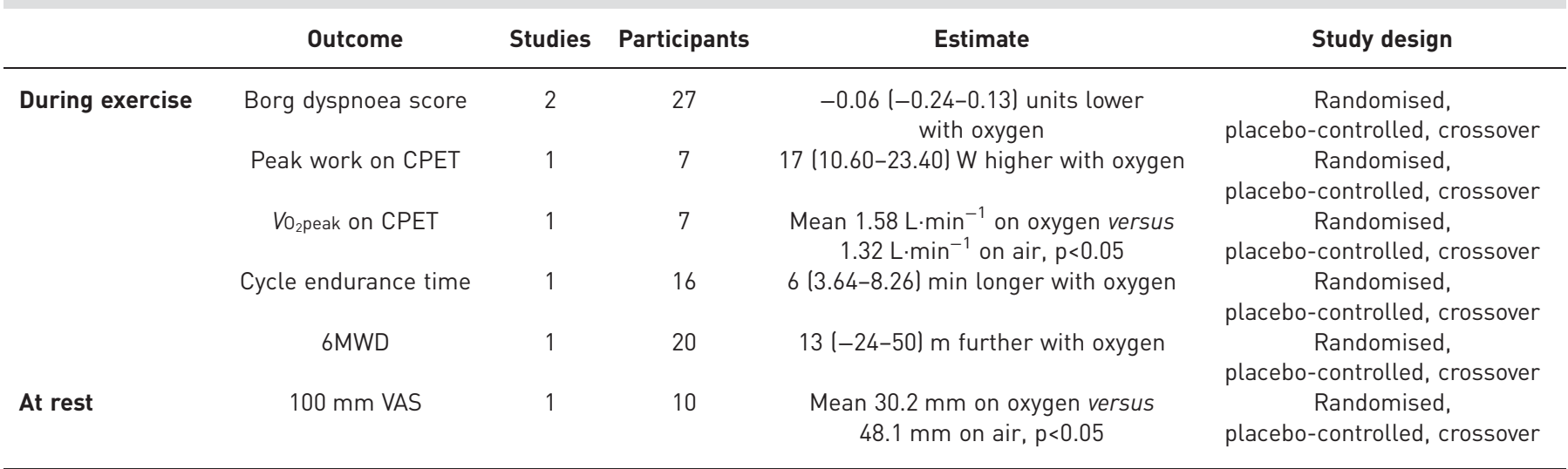

Data are presented as $\mathrm{n}$ or mean $(95 \% \mathrm{CI})$, unless otherwise stated. CPET: cardiopulmonary exercise test; $\mathrm{VO}_{2}$ peak: peak oxygen uptake; $6 \mathrm{MWD}$ : 6 min walk distance; VAS: visual analogue scale. 
No studies of LTOT reported outcomes for exercise capacity, mood disturbance or need for lung transplantation.

\section{Discussion}

This systematic review identified 12 studies that evaluated the impact of oxygen therapy in people with ILD. Short-term studies provided no consistent evidence that oxygen therapy administered during exercise reduced the primary outcome of dyspnoea. Randomised placebo-controlled trials support the positive impact of short-term oxygen therapy on exercise performance, although numbers of trials and participants are low (table 1). There were no randomised trials of LTOT. The quality of the body of evidence was limited by the retrospective nature of many studies, along with potential for selection bias and a lack of blinding for both participants and assessors.

There are many possible reasons that oxygen therapy might be prescribed for an individual with ILD who exhibits hypoxaemia at rest or during exertion, including reduction in dyspnoea, improved physical activity and functional independence, better HRQoL, prevention of secondary pulmonary hypertension and right heart failure and improved survival. We chose dyspnoea as the primary outcome of this review as it is an outcome of importance to patients that significantly impacts upon the quality of their lives [21, 22]. The mechanisms by which supplemental oxygen improves dyspnoea in hypoxaemic patients are not well understood but could include changes in chemoreceptor activation, the related reduction in respiratory drive and changes in breathing pattern, and/or stimulation of upper airway receptors by gas flow [23]. Despite a reasonable physiological rationale, we found no consistent evidence of an effect of short-term oxygen therapy on dyspnoea during exercise. One small but well-conducted study in acutely unwell hospitalised patients demonstrated a reduction in dyspnoea on VAS with oxygen at rest [10], which exceeded the minimal important difference [24]. However, it is not clear whether these results could be extrapolated to stable patients. In addition, the dyspnoea rating scales used during these short-term studies (Borg and VAS) reflect dyspnoea at a single point in time and are unlikely to capture the broader impact of dyspnoea on daily life. We found more consistent evidence for acute effects of short-term oxygen therapy on exercise performance for both maximal and endurance measures. While the only randomised trial to assess 6MWD [15] did not show improvements in this outcome that exceeded the minimum important difference [25], laboratory exercise measures from randomised, placebo-controlled crossover studies showed improvements with oxygen that were clinically important (table 1). Our findings differ from those of a recent Cochrane systematic review [5] on ambulatory and short-burst therapy that excluded these well conducted laboratory studies, and as a result they did not report the improvements in exercise capacity with oxygen seen in this review. Our results could inform discussions with patients regarding treatment aims; it is possible that patients using oxygen therapy on exertion could experience improvements in their physical capacity without noticeable improvement in symptoms. However, the absence of trials of ambulatory oxygen in ILD, where oxygen is used during the activities of daily living, makes it difficult to provide assurances that improved exercise capacity would be translated into improved physical activity levels in daily life.

International guidelines currently make a strong recommendation in favour of LTOT for people with IPF [26]. The guideline authors acknowledge the lack of studies supporting a survival benefit from LTOT, relying on indirect evidence from trials that include patients with obstructive lung disease. The two studies included in this review that compared patients using LTOT and those without LTOT were unable to identify benefits for survival or quality of life, and outcomes appeared worse in those treated with oxygen; however, these results are highly confounded, as those with more severe and progressive disease were more likely to receive LTOT $[18,19]$. An earlier Cochrane review identified one small RCT comparing LTOT to no treatment in patients with ILD [2]; however, this study has never been published in the peer-reviewed literature and as a result we did not include it. That study did not report a survival benefit of LTOT, so its exclusion would not have changed our findings. Given the degree of hypoxaemia experienced by many people with advanced ILD, along with established clinical practices for oxygen prescription, it is likely that LTOT will continue to be used widely in this group. However, care should be taken to carefully evaluate the benefits and costs to individuals. Recent qualitative studies have described the profound impact of LTOT on the lives of patients and carers $[22,27]$. While use of supplemental oxygen may improve confidence and enable patients to engage in meaningful activities [22], many also describe living in fear of running out of oxygen [22], and carers describe burdensome limitations to daily life [27]. These important impacts should be considered and discussed with patients at the time of LTOT prescription.

A strength of this study was the broad search criteria and robust methodology for assessing and incorporating studies evaluating different interventions and with varying risk of bias. As a result, this systematic review provides a comprehensive assessment of the impact of oxygen therapy in ILD. A major limitation to this review was the low quality of the included studies, many of which were at high risk of bias due to their retrospective or non-randomised nature (figure 2). Another limitation is the lack of 
studies evaluating other forms of oxygen therapy that are used in clinical practice, such as nocturnal oxygen therapy and short-burst oxygen therapy. We assessed a broad range of outcomes that are relevant to patients, but found no data for many, including mood disturbance, physical activity participation and need for transplantation. Other patient-important outcomes that we did not pre-specify in our protocol, including fatigue, cough and sleep quality [22], were not reported in any of the studies we included. These outcomes should be considered for future studies.

In conclusion, this systematic review has shown no consistent acute effects of short-term oxygen therapy on dyspnoea during exertion in ILD, although exercise capacity was increased. Studies reporting the use of LTOT in ILD are at high risk of bias and it was not possible to estimate its impact on survival. Future trials of oxygen therapy in ILD should evaluate whether the acute improvements in exercise capacity can be translated into improved daily physical activity and better HRQoL. In the absence of robust evidence of positive outcomes of oxygen therapy, patient perspectives on the benefits and costs will be important to inform treatment decisions.

\section{References}

1 Raghu G, Rochwerg B, Zhang Y, et al. An official ATS/ERS/JRS/ALAT clinical practice guideline: treatment of idiopathic pulmonary fibrosis. An update of the 2011 clinical practice guideline. Am J Respir Crit Care Med 2015; 192: e3-e19.

2 Crockett AJ, Cranston JM, Antic N. Domiciliary oxygen for interstitial lung disease. Cochrane Database Syst Rev 2001; 3: CD002883.

3 Nocturnal Oxygen Therapy Trial Group. Continuous or nocturnal oxygen therapy in hypoxemic chronic obstructive lung disease: a clinical trial. Ann Intern Med 1980; 93: 391-398.

4 Long term domiciliary oxygen therapy in chronic hypoxic cor pulmonale complicating chronic bronchitis and emphysema. Report of the Medical Research Council Working Party. Lancet 1981; 1: 681-686.

5 Sharp C, Adamali H, Millar AB. Ambulatory and short-burst oxygen for interstitial lung disease. Cochrane Database Syst Rev 2016; 7: CD011716.

6 Higgins JPT, Green S. Cochrane Handbook for Systematic Reviews of Interventions Version 5.1.0. www.handbook. cochrane.org Date last accessed: January 1, 2016. Date last updated March 2011.

7 Hirji KF, Fagerland MW. Calculating unreported confidence intervals for paired data. BMC Med Res Methodol 2011; 11: 66.

8 Anderson SD, Bye PT. Exercise testing in the evaluation of diffuse interstitial lung disease. Aust N Z J Med 1984; 14: $762-768$.

9 Bye PT, Anderson SD, Woolcock AJ, et al. Bicycle endurance performance of patients with interstitial lung disease breathing air and oxygen. Am Rev Respir Dis 1982; 126: 1005-1012.

10 Swinburn CR, Mould H, Stone TN, et al. Symptomatic benefit of supplemental oxygen in hypoxemic patients with chronic lung disease. Am Rev Respir Dis 1991; 143: 913-915.

11 Harris-Eze AO, Sridhar G, Clemens RE, et al. Oxygen improves maximal exercise performance in interstitial lung disease. Am J Respir Crit Care Med 1994; 150: 1616-1622.

12 Pouwels-Fry S, Pouwels S, Fournier C, et al. Effects of oxygen on exercise-induced increase of pulmonary arterial pressure in idiopathic pulmonary fibrosis. Sarcoidosis Vasc Diffuse Lung Dis 2008; 25: 133-139.

13 Visca D, Montgomery A, de Lauretis A, et al. Ambulatory oxygen in interstitial lung disease. Eur Respir J 2011; 38: 987-990.

14 Frank RC, Hicks S, Duck AM, et al. Ambulatory oxygen in idiopathic pulmonary fibrosis: of what benefit? Eur Respir J 2012; 40: 269-270.

15 Nishiyama O, Miyajima H, Fukai Y, et al. Effect of ambulatory oxygen on exertional dyspnea in IPF patients without resting hypoxemia. Respir Med 2013; 107: 1241-1246.

16 Crockett AJ, Alpers JH, Moss JR. Home oxygen therapy: an audit of survival. Aust N Z J Med 1991; 21: 217-221.

17 Chailleux E, Fauroux B, Binet F, et al. Predictors of survival in patients receiving domiciliary oxygen therapy or mechanical ventilation. A 10-year analysis of ANTADIR Observatory. Chest 1996; 109: 741-749.

18 Douglas WW, Ryu JH, Schroeder DR. Idiopathic pulmonary fibrosis: impact of oxygen and colchicine, prednisone, or no therapy on survival. Am J Respir Crit Care Med 2000; 161: 1172-1178.

19 Tomioka H, Imanaka K, Hashimoto K, et al. Health-related quality of life in patients with idiopathic pulmonary fibrosis - cross-sectional and longitudinal study. Intern Med 2007; 46: 1533-1542.

20 Cao M, Wamboldt FS, Brown KK, et al. Supplemental oxygen users with pulmonary fibrosis perceive greater dyspnea than oxygen non-users. Multidiscip Respir Med 2015; $10: 37$.

21 Swigris JJ, Gould MK, Wilson SR. Health-related quality of life among patients with idiopathic pulmonary fibrosis. Chest 2005; 127: 284-294.

22 Duck A, Spencer LG, Bailey S, et al. Perceptions, experiences and needs of patients with idiopathic pulmonary fibrosis. J Adv Nurs 2015; 71: 1055-1065.

23 Parshall MB, Schwartzstein RM, Adams L, et al. An official American Thoracic Society statement: update on the mechanisms, assessment, and management of dyspnea. Am J Respir Crit Care Med 2012; 185: 435-452.

24 Johnson MJ, Bland JM, Oxberry SG, et al. Clinically important differences in the intensity of chronic refractory breathlessness. J Pain Symptom Manage 2013; 46: 957-963.

25 Holland AE, Spruit MA, Troosters T, et al. An official European Respiratory Society/American Thoracic Society technical standard: field walking tests in chronic respiratory disease. Eur Respir J 2014; 44: 1428-1446.

26 Raghu G, Collard HR, Egan JJ, et al. An official ATS/ERS/JRS/ALAT statement: idiopathic pulmonary fibrosis: evidence-based guidelines for diagnosis and management. Am J Respir Crit Care Med 2011; 183: 788-824.

27 Belkin A, Albright K, Swigris JJ. A qualitative study of informal caregivers' perspectives on the effects of idiopathic pulmonary fibrosis. BMJ Open Respir Res 2014; 1: e000007. 